\title{
Spatial Writing: A New Mode of Architectural Heritage Representation
}

\author{
SEHER ERDOĞAN FORD
}

Temple University

\begin{abstract}
Modeling, as an instrument of architectural design, delineates boundaries for new ideas and establishes a framework for approaching the unknown. Those boundaries, however, often leave out elements of the design process and the larger context of the project. This is a particularly critical issue when dealing with architectural heritage sites with complex histories and multiple identities. As part of a larger research project dealing with the representation of cultural heritage, in this paper I pose the question: How can digital modeling embody the intangible dimensions of architectural production?
\end{abstract}

\section{INTRODUCTION}

The digital model as an embodiment of the intangible dimensions of architecture suggests a dichotomy: how to relate quantifiable and "discrete" components of built work to the qualitative and ambiguous aspects that help make it. This duality is in fact at the heart of many transdisciplinary inquiries conducted under the umbrella of "digital humanities," a field that utilizes robust computational methods to pursue interpretive, humanities-oriented research.

Digital humanities encompass such fields as archeology, anthropology, geography and history, and employs a multitude of cross-disciplinary methodologies. One particular strain of digital humanities project deals with 3D digital reconstructions of heritage sites and utilizes the virtual model to reveal unseen or unknowable "layers" of architecture.

I begin the argument by introducing the notion of spatial writing through several types of digital humanities projects of multidisciplinary aspirations. And I continue to discuss in detail one such project dealing with a heritage site in Istanbul, Turkey. I illustrate how it functions as a spatial writing project and why virtual reality (VR) is an appropriate medium for projects similar in nature. Ultimately, I articulate the idea of spatial writing as a new mode of architectural representation that warrants deeper examination by researchers employing the design of the virtual environment for generative purposes and designers engaging in research-based practice.

VIRTUAL ENVIRONMENTS IN THE DIGITAL HUMANITIES Digital Humanities, as a field, pursues humanities-oriented questions by use of robust computational methods. This approach sits at the intersection of quantitative and qualitative thinking and transdisciplinary methodologies. With continually transforming technologies, so are the defining boundaries and applicable media of digital humanities projects. Current literature on the field identifies the following primary genres of projects: data mining and text analysis, cultural analytics, network analysis, GIS mapping, and virtual reconstructions.1

This paper deals with the close examination of virtual reconstructions through the lens of architectural representation. Many virtual reconstructions cover archeological or architectural sites of historical significance. Created by multidisciplinary teams, these digital constructions typically use the 3D model as an underlay for other kinds of information, relating for instance such aspects as programmatic rituals, cultural traditions, sensory experiences and temporal shifts. As virtual environments, they exist on a range of 3D modeling platforms and reach a wider audience on the web via screens or virtual reality headsets.

What are some defining qualities of digital humanities projects? At their core is the notion that knowledge production is never a value-neutral endeavor and therefore all visualization, as far as it represents the world in some form and not another, is coded with the biases of its authors and their specific cultural contexts. Furthermore, digital humanities projects make this subjective aspect of modeling transparent by exhibiting the data along with the ontological framework that shaped its curation. The visualization of the virtual world is accompanied by scholarly information that helped build it; furthermore, it remains dynamic. The resultant has also been referred to as information-rich virtual environments by some researchers. 2

\section{SPATIAL WRITING AS A MODE OF REPRESENTATION}

In this paper, I argue that these types of projects, by virtue of their intent to hold heterogenous content within spatial constructs, constitute examples of a new mode of architectural representation: spatial writing. This is a term that the visual theorist Johanna Drucker introduces in her 2014 book Graphesis: Visual Forms of Knowledge Production. In it, Drucker builds an argument for the distinction between visualizations that are "representations of information already known and those that are knowledge generators capable of creating new information through their use." 3 Drucker defines spatial writing projects as dynamic repositories of heterogeneous content, creating "diagrammatic, associative, and constellationary digital spaces" that implement the 3D model as a device for new scholarship.

Spatial writing assumes the active participation of the audience as researcher, writer, reader and essentially as 


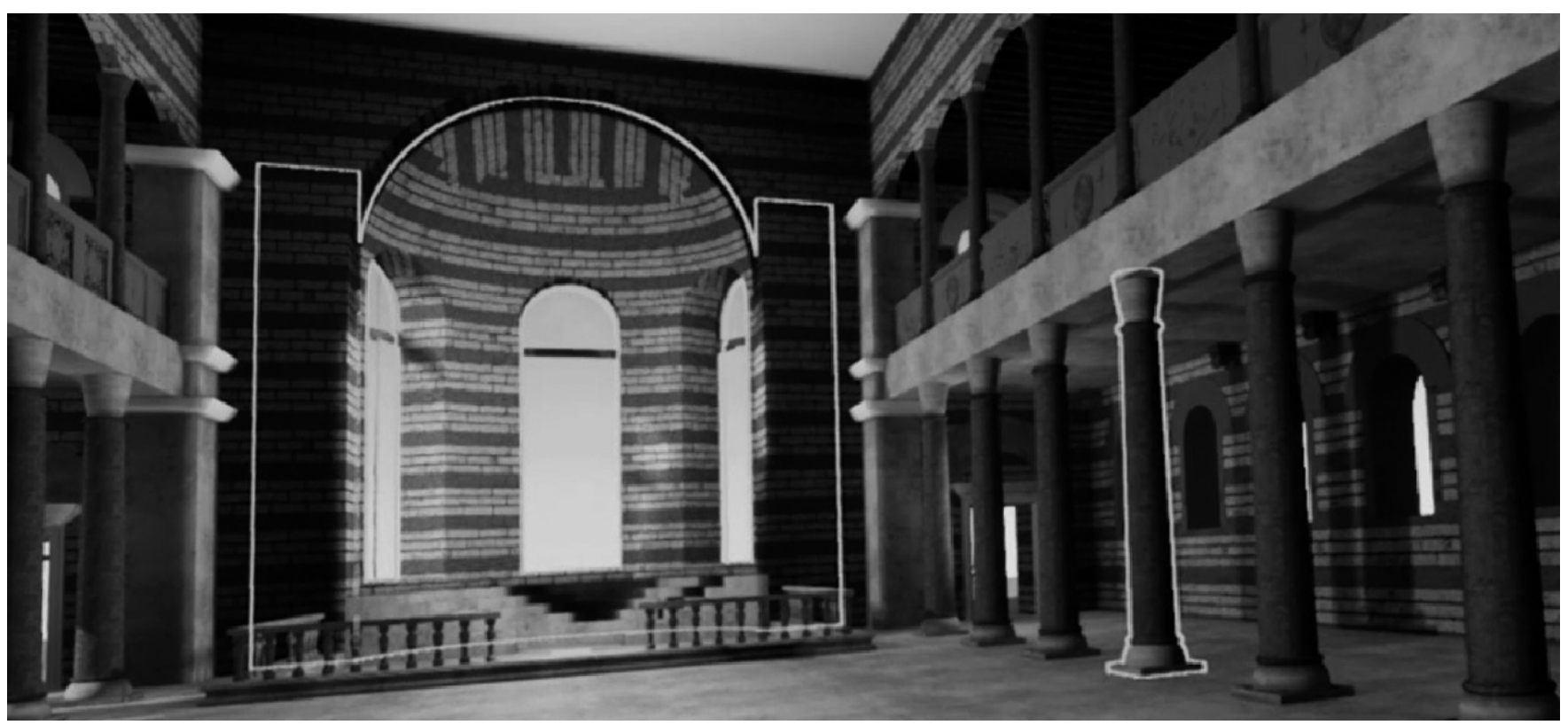

Figure 1: Overall view of the virtual model interior with the annotated elements highlighted.

interactor, erasing the "edge" of the work and blending the multiple "hands" that contribute to knowledge generation. As a consequence, it describes a research practice more than a predefined form of outcome. Drucker's conception of this type of digital space states three qualities-diagrammatic, associative, and constellationary - that set apart spatial writing from other modes of representation.4 As diagrammatic frameworks, their information structures are designed to produce a deliberate meaning. For this, a certain level of abstraction is necessary to clearly convey the fragmented and at times uncertain nature of the information there may be involved.

This mode of representation is generative as far as it requires the researcher/interactor to actively create associations between things and ideas that may exist in the form of textual, visual, and possibly multi-sensory data. These connections function as the sequential operations of an interpretive process that generates a different narrative with each researcher's intent, creating layers of meaning by associating multiple narratives and producing collective authorship. Such a reliance on associative thinking and its creative possibilities is a distinctive and important feature of humanities-oriented research.

As a constellationary formation, spatial writing projects mimic a curatorial process in which disparate elements come together to form recognizable and/or new patterns. These patterns depend on the perspective of the observer, both in the physical as well as the temporal sense, relative to the set of data. As a consequence of spatially juxtaposing these elements (and similarly to how constellations form), elements appear "together" although they may be at a variety of distances from one another and from the observer. New and unlikely connections are made, and novel patterns emerge. The researcher as writer/reader can observe the resultant gestalt of the patterns (to borrow a term from visual language) and draw new conclusions and meanings from these curatorial experiments. Spatial writing projects build narratives across physical spaces and temporal continuums.

What does spatial writing in virtual reality look like? Furthermore, what does it achieve that other digital spaces cannot? As a medium, VR offers two unique qualities that enhance the practice of spatial writing. First, self-navigation within the digital space engenders a sense of agency in the user. This is both an actual ability to probe and to discover the virtual environment as well as a psychological shift in the participation of the user in the "making" of the environment. The 3D model is a device for the interactor to engage with and activate as a tool for research. As such, the model is not a sequence of images and volumes, but more an indexical environment that unfolds in different ways for different users and their research interests, literal and figurative perspectives. In addition, the immersive nature of VR clarifies and in fact simplifies the use of architectural constructs in digital spaces. The 3D model need not be limited to a metaphor for organization but can act as an actual armature for information.

In this paper I consider what form(s) the spatial writing of architecture can take in VR-its affordances that set it apart from conventional modes of visual representation. In order to elaborate on the specific ways in which spatial writing can be applied to the study of architectural heritage sites, I discuss the design of an initial prototype built with technical assistance from Chris McAdams and Julie Kress on the VR platform UnrealEngine, to create an indexical virtual environment. 


\section{THE ARCHITECTURAL HERITAGE SITE}

The heritage site in question is the remnants of the Church of Stoudius (later known as the Mosque of Imrahor) in present-day Istanbul, Turkey. The church was a highly prominent center of cultural and religious life in the Byzantine Empire and was converted to a mosque by the Ottomans upon the conquest of Constantinople. The oldest surviving religious structure in Istanbul, the ruin has been protected as a heritage site by the Turkish state but was slated for an extensive (and potentially destructive) reconstruction project to convert it to a functional mosque in 2015. Since the announcement of this controversial initiative, the site has been strictly inaccessible. Given these circumstances, there is an urgency to develop a digital project capturing the architectural heritage of the building as it transformed over time under different political/programmatic agendas.

To portray the complex history of this architectural artifact, I conceived the digital model as a discrete repository of content as well as a node within a larger network. It is a repository because elements within the 3D model hold pertinent information about the building's history. But it is not a selfcontained construct as much of the information on the site is connected to external sources that offer broader avenues for research. Therefore, the content embedded within the model creates access points into deeper and broader contexts.

Archival investigations highlight the uncertainty of some data and the incompleteness of historical records, introducing ambiguity as a challenge to be grappled with in the modeling process. In response, the 3D model does not reflect a definitive reproduction of what the building was, but an interpretive process of what it may have been. As a consequence, the model is freed from the pursuit of photorealistic rendition and functions as an instrument of the research process with its own logic of graphic communication and language, carried out through spatial writing.

\section{THE DESIGN OF THE VIRTUAL ENVIRONMENT}

The spatial writing project makes the pertinent references accessible in the form of multimedia annotations in VR. Annotations, in writing, offer the reader a deeper view or a tangential approach without interfering with the primary space of the text and or convoluting the narrative. In this project, we explore ways in which the multimedia annotations within a 3D model can function in a similar way by overlaying onto diagrammatic structures, suggesting associations, and forming constellationary patterns - in conformance with Drucker's description of spatial writing.

What are some of the attributes of annotation in architectural representation? This question opens up two parallel lines of inquiry: one is in regard to the curatorial intent, in other words what content gets embedded within the annotations, and the second has to do with the formal design of how annotations are experienced in VR.

A unique aspect of spatial writing is its facility to intertwine the textual and the visual, departing from the primacy that traditional scholarship places on the printed word. This is crucial to the representation of the Stoudius site since much of what we know about it is drawn from heterogeneous material, ranging from archival drawings, photographs, and prints to texts authored by a variety of sources. The source material is made further crucial by the fact that the site is closed to visitors and any type of field investigation including photography and scanning.
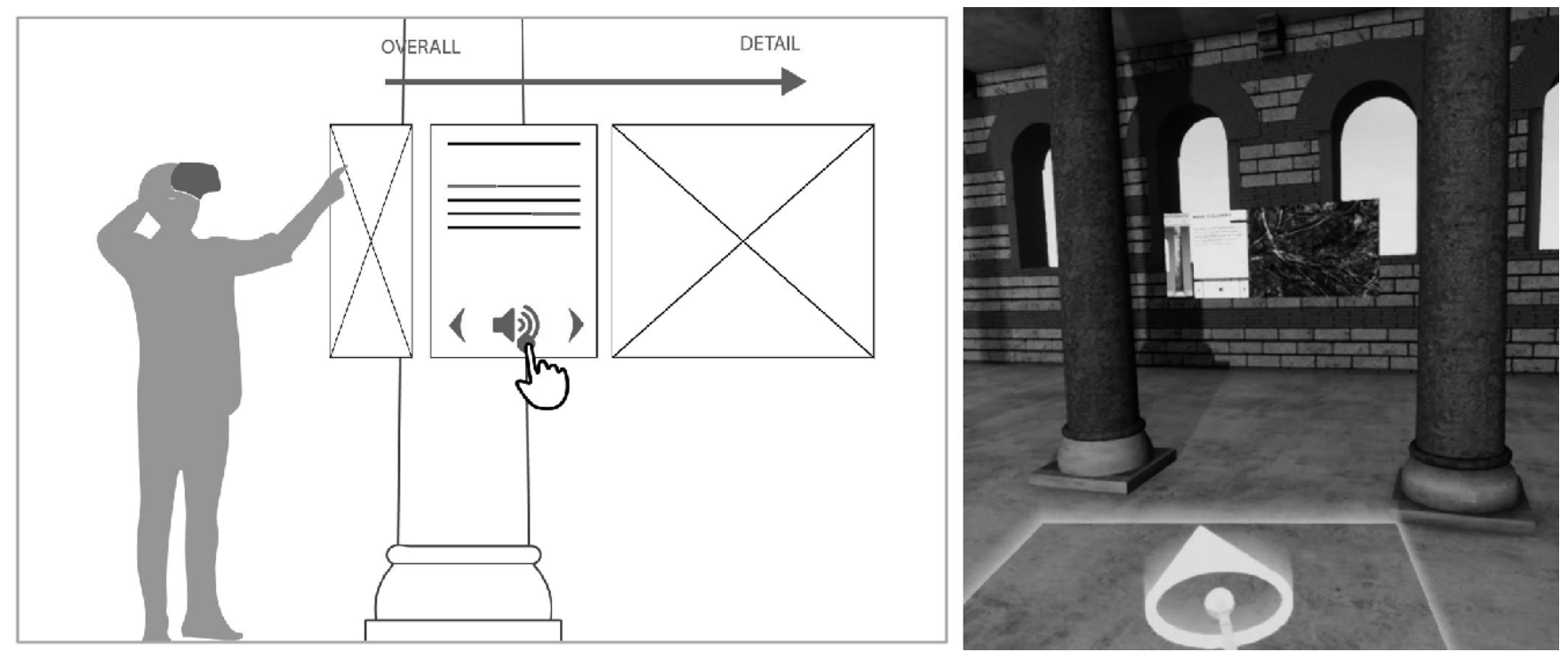

Figure 2: Diagram outlining the basic organization of the widgets (left) and the view of the activated annotation in VR (right). 
In addition to what already exists in the archives, designing directly with the new medium of virtual reality and observing the persistent limitations of text legibility and image resolution suggests an opportunity to include yet another type of content: audio. This begs further exploration, but our initial strategy for incorporating sound was to counteract the difficulty of reading long texts by offering in-depth narratives via sound recordings.

As Drucker asserts in Graphesis, the structure in which the content is organized influences the semantic reading of it. Portraying the continuous lifecycle of the building in flux and in relation to a multitude of contexts has been central to the conceptual framework of the project. With this in mind, I borrowed methodology from anthropology and archeology in regard to analyzing cultural artifacts. Commonly referred to in French as "chaîne opératoire," the method traces the chain of operations due to social acts over the life of an object, encompassing the extraction of its raw materials to its tooling and application in construction to functional use and disuse to its decay and regeneration. Ultimately, this longer view on the making of a building offers a new perspective on architectural production.

Prompted by the question of what form an architectural chaîne opératoire can take, I tested several studies for exploring ways of conveying the multivalent nature of architectural production. What materials were used in the making of the columns? Where were they sourced? How were they fabricated to create the capitals? How were they altered after the series of renovations the building has gone through? Such questions suggested a form of representation that traces two types of scale, along both the physical and the temporal axes. While both are linear, the change in physical scale involves looking more closely at details or zooming out to see larger patterns, and the temporal scale traces time and its material registrations on the building as textures or changes in massing. They are distinctly different modes of inspecting the physical, material qualities of the built environment, with possibilities for intersection.

Representation of multiple scales of physicality is a particularly loaded proposal for virtual reconstructions that provide an immersive experience. As Paul Emmons in his essay "Drawn to Scale: The Imaginative Inhabitation of Architectural Drawings" argues, 1:1 scale is not the ultimate "desire" (or goal) motivating any act of drawing. Scaled drawings do not limit accuracy, but in fact they facilitate comprehension of intent and analysis of applicability and further, in Emmon's words, explicate the "nature of architectural conception." 5 Virtual environments promise full-scale representation but in practice risk scalelessness. The risk here is that the designer or the interactor views the 3D space as a contained object rather than engaging in "imaginative inhabitation" that conjures "possible worlds." 6 The ability to be able to project oneself into the space of the drawing is critical for this imagination to be activated but also to gain a proportional understanding of the construct being created. This is also the opening where an interpretive reading might take place and where the two "axes" of physical and temporal might intersect.

This line of thinking can be translated from 2D drawings to 3D space accessed in VR. However, this change in medium requires careful attention to the notion of "imaginative inhabitation," especially since one may assume it to be a non-issue since the viewer can actually inhabit the space. While the perception of scale may be much more palpable in VR than in drawings, the danger of scalelessness is in fact greater in VR. The surfaces and volumes and details rendered at full-scale and seemingly coexisting in the same world as the human body curtail discursive possibilities and potential interpretations. Therefore, we must probe the question as to how VR can engender a more imaginative space within the framework of digital architectural representation.

I argue that the need for scaled representation is not eliminated in VR, but on the contrary, all the more amplified. The dynamic relationship between the visitor's body moving within the boundaries of the virtual space and the visitor's mind imagining beyond those same boundaries to reach other potentialities can be facilitated by drawings. The VR visitor must actively engage with the spatial construct to acquire a level of "comprehension of drawn worlds possible" and not be confined to a singular virtual space.7 Therefore, in this project we experiment with how digital space can reinforce a bodily sense of scale by including drawings in direct juxtaposition with the full-scale architectural elements experienced in VR.

If these boundaries open up, what is the nature of the relationship between the visitor in virtual space and the outside, real world? Media scholar Pierre Levy posits that in the virtual world of texts, images, sounds and even tactile qualities, the "user" becomes an interactor and thereby an agent of content creation. 8 By activating the annotations through discovery, making selections and creating potentially synthetic connections with potentially new meanings, the interactor interprets and transforms the model each time they "visit." Levy also goes on to state "every act of reading is a potential act of writing, contributing to collective knowledge."9The interactor as writer is not a singular agent but part of a fabric of knowledge creation. As the model is a porous entity offering access to multiple external sources and databases--other interpretations--it is situated at the intersection of other lines of inquiry. The curated content offers entry points into other external sources, allowing the visitor in VR, as "interactor," to weave multiple narratives. Spatial writing is not an isolated, singular activity but a collective task. This is a powerful and highly suggestive notion that would benefit from further consideration of what the collaborative act of drawing and making can look like in contemporary architectural scholarship ad practice. 

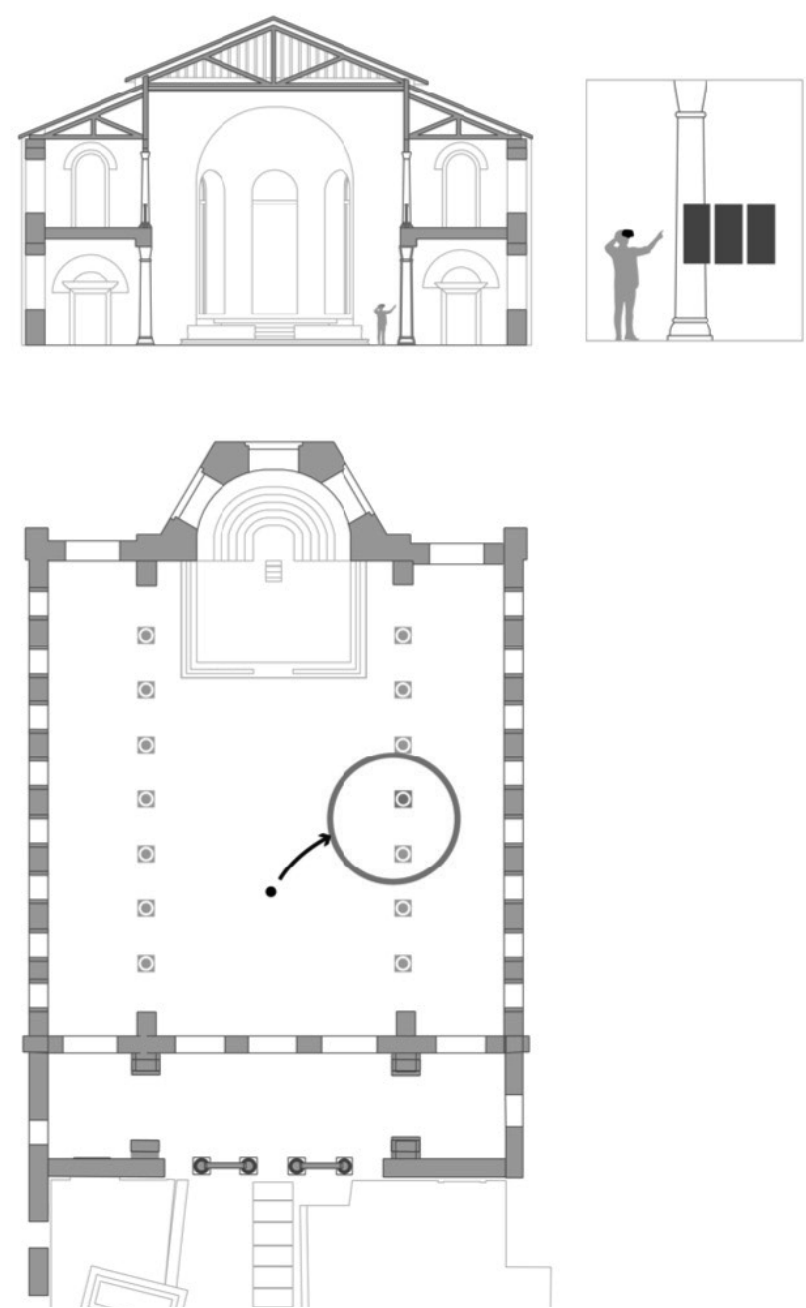

Figure 3: Diagrammatic drawings indicating the placement of the annotations relative to the visitor's body.

\section{THE DESIGN OF THE INTERACTIVE EXPERIENCE}

Given this framework, we delved into the specific aspects of the visitor's interactive experience within the virtual environment. Initial studies focused on the conceptual scaffolding for placing annotations within the model. From a technical perspective, the annotations linked to specific components within the model were made possible by assets called "widgets" in the Unreal VR platform.

We examined the range of strategies as far as where annotations "lived." Three distinct options emerged. First, we considered layering the widgets on the "head-up display" (HUD) that exists in a separate picture plane dissociated from the 3D model but visible in the viewer's field of vision. In this scenario, the navigator would view the annotative content not within the model but in an interstitial space often reserved for interactive elements of the interface. This separation of the widget content from the model and its placement in a layer of the digital space where mediation is explicit proved to break the direct associations we wanted to create with the 3D elements and the supporting scholarly content. A different strategy was to embed the annotations within the model, either associated with a specific 3D element or a set of coordinates within the model. Initially we tested the sequence in which the widget is anchored in a specific location within the mode, at a spot where the virtual visitor may stop and view the element in question. Designating a viewing point made sense with one annotation but quickly exposed its flaws when considering the deployment of a multitude of widgets associated with a variety of elements. Furthermore, from an experiential point of view, fixing the information at prescribed points for the viewer to stand on suggested a forced imposition of a perspectival experience and counteracted the fluid nature of the immersive medium. Finally, upon further experimentation, the annotative content being fixed to an architectural element and "discoverable" through self-navigation made sense with the generative function the model served.

From an experiential point of view, the annotated model offers an intuitive user interface. As the visitor explores the virtual environment, certain elements of the building are "highlighted" with dynamic, colorful outlines that signal interactivity. As the visitor approaches one of these elements and reaches a certain proximity, we designed the widget to appear and disappear when the person walks away. This way, the visitor visually recognizes opportunities for further exploration but may decide to activate these layers in their own time.

We tested the annotative widgets in VR as both 2D and 3D elements. To counteract the potentially flat reading of the widgets, and to create a more spatial application, we considered the body's interaction with the widget as well as the form of the widget.

At first we studied how the visitor's movement through the space might activate the widgets. Instead of interactive elements such as an "on/off" button, we wanted to incorporate the movement and proximity of the visitor as a factor in what content would appear. Our reasoning was that this reinforced the idea that the content was embedded within the 3D model and was accessible with spatial triggers, as opposed to existing in a separate, abstract repository outside of the model space. With this idea in mind, we also determined the appropriate dimensions of proximity and area that would function as boundaries or thresholds for the content to be activated. These invisible boundaries created a layer of editorial content that was dynamic by virtue of its ability to be updated as well as its responsiveness to user activity. Other parts of the model remained static. An additional component that became activated in response to visitors' movement was the audio narratives that were also specifically associated with certain boundaries around an architectural element. The audio was 

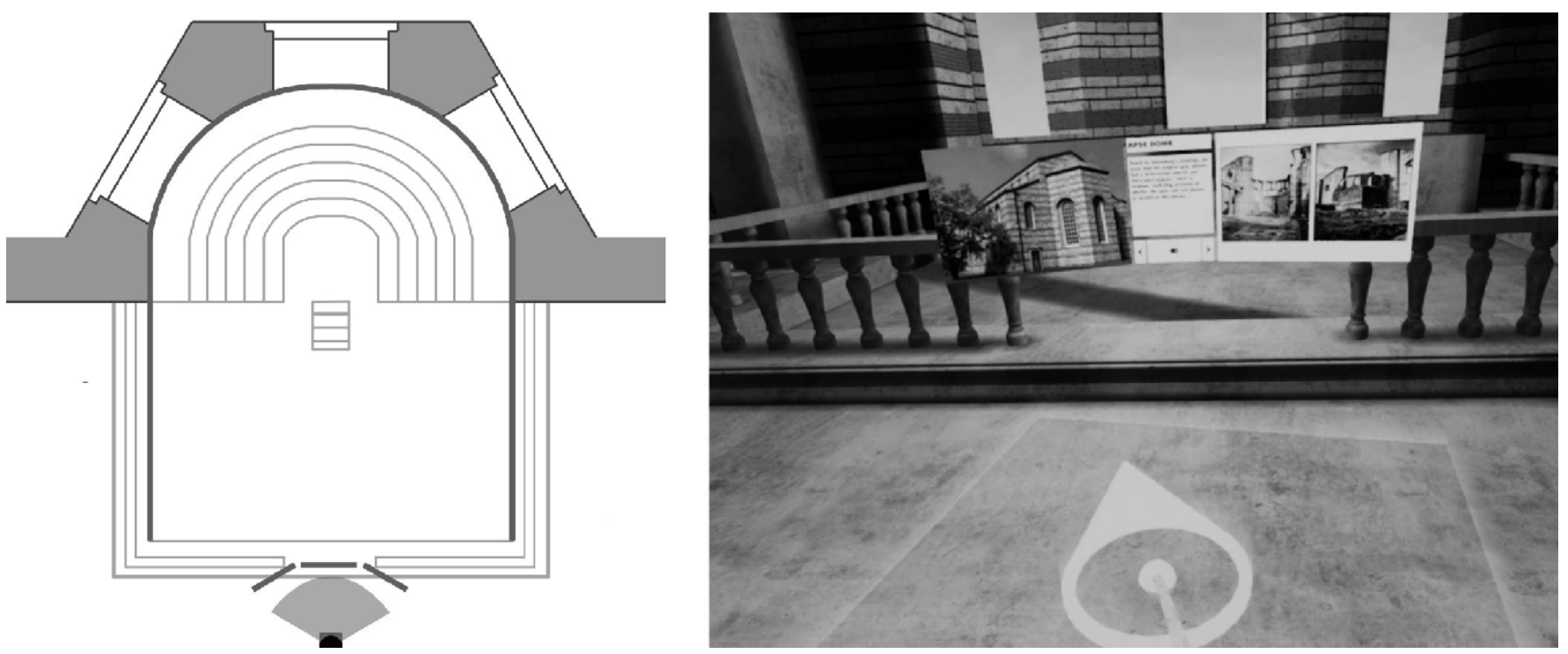

Figure 4: The plan diagram outlining the three-dimensional design of the annotative widget (left) and the view of the activated annotation in VR (right).

"spatialized" by coding it such that it sounded far away as the navigator walked away from the source, reinforcing the desired effect of keeping textual, visual, and auditory content closely associated with the architectural element in question.

As far as the spatial experience of the annotations, our primary motivation was to design the widgets such that they did not register as flat elements or "plaques," reminiscent of analog components of exhibition design, and therefore not native to the immersive medium. In order to achieve this, while designing the widgets, which are essentially flat, we considered their relationship to the body and the annotated architectural element in both plan and elevation to trigger nuanced cues of three-dimensionality. Parameters such as the size of the widget, its distance from the anchoring object, its elevation from the ground, and its profile in plan involved crucial adjustments. While this still requires exploration and development, as an initial attempt, we employed angular arrangements in plan to suggest spatiality, responding to the approach of the body through space toward the element in question. Other considerations such as density, distance and visual cues for increased legibility, as well as an intuitive interactive experience, have been factors influencing certain decisions throughout the design process, and are of particular interest in looking forward to the future phases of the project.

As scalable, discrete objects with specific functions encoded, widgets allowed us to design the layout and the graphic qualities of the annotations and deploy them across the VR model as needed. These designs were developed as wireframe studies, through which we examined various options for arrangements of textual and visual blocks. Subsequently, we tested various configurations of text in VR environment in terms of ease of use, intuitive interactivity, and legibility, reaching conclusions about acceptable ranges in terms of width and length for the text block such that the navigator would be able to read without shifting their range of view. In addition, we tested time durations for reading to ensure the experience not be cumbersome. In parallel, we looked at how to organize the visual material around text blocks. Common forms such as slide shows, decks, and grids were some of the few options we considered that would feel most intuitive for the navigator. Given our decision to limit the image quantity in keeping with the curatorial choices, options where the visitor viewed pairs of images in sequence made more sense. As far as the visual content's spatial relationship to the verbal information, our tests showed that locating the text frontally and the images on either side of the block felt easiest to use and reinforced the curatorial message. On the left side of the text, we located a static element (a photorealistic rendering of the architectural element of interest), and to the right of the text sat dynamic elements containing single or pairs of images that captured the physical qualities of the architectural element at varying scales and over time. Furthermore, this arrangement reinforced the curatorial intent shaped by the chaîne opératoire method by anchoring a static reference image whose transformation would register along the temporal and physical scales.

As nodes of a larger network, annotations also contained access points to external sources in the form of hyperlinks embedded within the text. The choice of which words were to be identified as keywords for further research was based on the available literature, significant elements of the architecture, and important historical dates. We used the easily recognizable strategy of color differentiation to visually separate these terms. As for the functionality of hyperlinks, there were two available options. One was to create a bit of code, which would trigger 
the opening a new tab in the web browser with each click on the hyperlink. The other, and conceptually more desirable, option for this project was to integrate the web search within VR. The latter functionality is possible but in the very early phases of its development, yielding cumbersome user experience and "glitchy" functionality. Therefore, this aspect of the hyperlink experience remains to be further developed.

\section{CONCLUSION}

Digital models accessed in virtual reality offer new modes of representation in which visualizations go beyond reproductions of static knowledge and facilitate interpretive readings and discovery. This is possible by employing a "spatial writing" practice introduced as a notion in the Digital Humanities and in which visual, textual, and auditory content is intertwined to construct complex stories that are otherwise seldom represented and convey messages that are typically omitted from conventional modes of architectural representation. Engaging in the interdisciplinary scholarly practice of spatial writing and implementing the large suite of technologies associated with it can transform and advance architectural modeling as implemented by designers and scholars, but also help reimagine the disciplinary boundaries of visual and multisensory representation.

\section{ENDNOTES}

1. Anne Burdick, Johanna Drucker, Peter Lunenfeld, Todd Presner, and Jeffrey Schnapp. Digital_Humanities (Cambridge, MA: MIT Press, 2016), 12.

2. Doug A. Bowman, Chris North, Jian Chen, Nicholas F. Polys, Pardha S. Pyla, and Umur Yilmaz, "Information-Rich Virtual Environments: Theory, Tools, Reality Software and Technology, (2003): 81-90.

3. Johanna Drucker, Graphesis Visual Forms of Knowledge Production (Cambridge, Mass. : Harvard University Press, 2014), 65.

4. Drucker, Graphesis, 183.

5. Paul Emmons, "Drawn to Scale: The Imaginative Inhabitation of Architectural Drawings." In From Models to Drawings: Imagination and Representation in Architecture. ed. Marco Frascari, Jonathan Hale and Bradley Starkey (London New York: Routledge, 2007), 64

6. Emmons, Drawn to Scale, 71.

7. Emmons, Drawn to Scale, 73.

8. Pierre Levy. "Toward a Superlanguage." Plenary Speech, ISEA, Helsinki, Finland, August 22, 1994. 